Cahiers $d u$ MONDE RUSSE

\section{Cahiers du monde russe}

Russie - Empire russe - Union soviétique et États indépendants

$62 / 4 \mid 2021$

Varia

\title{
Carsten GOEHRKE, Unter dem Schirm der göttlichen Weisheit Geschichte und Lebenswelten des Stadtstaates Groß-Nowgorod
}

\section{Pavel Lukin}

\section{OpenEdition}

Journals

Édition électronique

URL : https://journals.openedition.org/monderusse/12704

DOI : 10.4000/monderusse. 12704

ISSN : 1777-5388

Éditeur

Éditions de l'EHESS

\section{Édition imprimée}

Date de publication : 1 décembre 2021

Pagination : 658-666

ISBN : 978-2-7132-2895-7

ISSN : $1252-6576$

Référence électronique

Pavel Lukin, «Carsten GOEHRKE, Unter dem Schirm der göttlichen Weisheit Geschichte und Lebenswelten des Stadtstaates Groß-Nowgorod», Cahiers du monde russe [Онлайн], 62/4 | 2021, Выложить онлайн 01 décembre 2021, Наводить справки в 03 septembre 2022. URL: http://journals. openedition.org/ monderusse/12704; DOI: https://doi.org/10.4000/monderusse.12704

Ce document a été généré automatiquement le 3 septembre 2022.

All rights reserved 


\title{
Carsten GOEHRKE, Unter dem Schirm der göttlichen Weisheit Geschichte und Lebenswelten des Stadtstaates Groß- Nowgorod
}

\author{
Pavel Lukin
}

\section{RÉFÉRENCE}

Carsten GOEHRKE, Unter dem Schirm der göttlichen Weisheit Geschichte und Lebenswelten des Stadtstaates Groß-Nowgorod, Zürich : Chronos, 2020, 560 p.

РАБОТА ПОДГОТОВЛЕНА В РАМКАХ ГРАНТА, ПРЕДОСТАВЛЕННОГО МИНИСТЕРСТВОМ НАУКИ И ВЫСШЕГО ОБРАЗОВАНИЯ РОССИЙСКОЙ ФЕДЕРАЦИИ (№ СОГЛАШЕНИЯ О ПРЕДОСТАВЛЕНИИ ГРАНТА: 075-15-2020-908).

1 КНИГА ВИДНОГО НЕМЕЦКОГО ИСТОРИКА-СЛАВИСТА КАРСТЕНА ГЁРКЕ, МНОГО ЛЕТ РАБОТАВШЕГО В ЦЮРИХСКОМ УНИВЕРСИТЕТЕ В ШВЕЙЦАРИИ, ПОСВЯЩЕНА, ПОЖАЛУЙ, ЦЕНТРАЛЬНОЙ ТЕМЕ ЕГО ИССЛЕДОВАНИЙ - ИСТОРИИ НОВГОРОДА. ЕМУ ПРИНАДЛЕЖИТ РЯД ВЕСЬМА ЦЕННЫХ РАБОТ В ЭТОЙ ОБЛАСТИ, И МОЖНО ТОЛЬКО ПОСЕТОВАТЬ, ЧТО ОНИ НЕДОСТАТОЧНО (НЕЗАСЛУЖЕННО!) ИЗВЕСТНЫ В РОССИЙСКОЙ ИСТОРИОГРАФИИ, А НЕКОТОРЫЕ ВЫВОДЫ, СДЕЛАННЫЕ К. ГЁРКЕ, ПОТОМ ПОВТОРЯЛИСЬ ДРУГИМИ АВТОРАМИ, НЕ ЗНАВШИМИ ТРУДОВ ПРЕДШЕСТВЕННИКА. РАБОТЫ К. ГЁРКЕ ПО ИСТОРИИ НОВГОРОДА, МЕЖДУ ТЕМ, БУДУЧИ ВЫПОЛНЕНЫ В ПОЧТЕННОЙ ТРАДИЦИИ НЕМЕЦКОЙ Verfassungsgeschichte, НО В ТО ЖЕ ВРЕМЯ НЕ ЧУЖДЫЕ ВЕЯНИЯМ НОВЫХ ПОДХОДОВ В ОБЛАСТИ СОЦИАЛЬНО-ПОЛИТИЧЕСКОЙ ИСТОРИИ, ОТЛИЧАЮТСЯ СЛЕДУЮЩИМИ, ГЛАВНЫМИ, НА МОЙ ВЗГЛЯД, ПОЛОЖИТЕЛЬНЫМИ ЧЕРТАМИ. ВО-ПЕРВЫХ, ЭТО СТРЕМЛЕНИЕ К ПОЛНОМУ ПО ВОЗМОЖНОСТИ ОХВАТУ ВСЕГО КОМПЛЕКСА ИМЕЮЩИХСЯ ИСТОЧНИКОВ И ИХ ТЩАТЕЛЬНЫЙ И ОБЪЕКТИВНЫЙ РАЗБОР. ВО-ВТОРЫХ, ЭТО ПРЕДПОЧТЕНИЕ ФАКТОВ ТЕОРИЯМ, СКОЛЬ УГОДНО КРАСИВЫМ. В-ТРЕТЬИХ, ЭТО ТО ЧТО 
МОЖНО НАЗВАТЬ ИНТЕЛЛЕКТУАЛЬНОЙ И АКАДЕМИЧЕСКОЙ ЧЕСТНОСТЬЮ: ИСТОРИК, ВЗВЕШИВАЯ АРГУМЕНТЫ, ГОТОВ СОГЛАСИТЬСЯ С САМЫМИ УБЕДИТЕЛЬНЫМИ ИЗ НИХ, ДАЖЕ ТЕМИ, КОТОРЫЕ НЕ ВПОЛНЕ СООТВЕТСТВУЮТ ЕГО КОНЦЕПЦИИ. НАКОНЕЦ, ПРОФЕССОР ГЁРКЕ ПРЕКРАСНО ЗНАЕТ РОССИЙСКУЮ ЛИТЕРАТУРУ О НОВГОРОДЕ И ОТНОСИТСЯ К ДОСТИЖЕНИЯМ РУССКИХ УЧЁНЫХ ОЧЕНЬ ВНИМАТЕЛЬНО И УВАЖИТЕЛЬНО. ВСЕ ЭТИ ДОСТОИНСТВА ЯВЛЕНЫ И В РЕЦЕНЗИРУЕМОЙ КНИГЕ, КОТОРАЯ ПРЕДСТАВЛЯЕТ СОБОЙ ПОДРОБНЫЙ ОЧЕРК РАЗНЫХ АСПЕКТОВ ИСТОРИИ НОВГОРОДА. ПОСЛЕ УЖЕ В ЗНАЧИТЕЛЬНОЙ МЕРЕ УСТАРЕВШЕГО И ПО НЕОБХОДИМОСТИ НЕПОЛНОГО ОБЗОРА ТОГО ЖЕ АВТОРА В Handbuch der Geschichte Russlands ${ }^{1}$ ЭТО ЕДИНСТВЕННЫЙ НА СЕГОДНЯШНИЙ ДЕНЬ НАУЧНЫЙ ОБЗОР ИСТОРИИ СРЕДНЕВЕКОВОГО НОВГОРОДА НА НЕМЕЦКОМ ЯЗЫКЕ.

2 КНИГА СОСТОИТ ИЗ ТРЁХ РАЗДЕЛОВ: ВВЕДЕНИЯ (ХАРАКТЕРИСТИКА ИСТОЧНИКОВ, ИСТОРИОГРАФИИ И НОВГОРОДСКОГО ДИАЛЕКТА), ОСНОВНОЙ ЧАСТИ И ЗАКЛЮЧЕНИЯ, В КОТОРОМ РАССМАТРИВАЕТСЯ ПОСТ-РЕСПУБЛИКАНСКАЯ ИСТОРИЯ НОВГОРОДА (ТАК СКАЗАТЬ, Novgorod après Novgorod) И ДЕЛАЕТСЯ ПОПЫТКА ОСМЫСЛЕНИЯ НОВГОРОДА И ЕГО «ГОСУДАРСТВЕННОЙ МОДЕЛИ» (Staatsmodell) В ШИРОКОМ КОНТЕКСТЕ. В ТО ЖЕ ВРЕМЯ, АВТОР ЯВНО ОБРАЩАЕТСЯ - ХОТЯ И СПЕЦИАЛЬНО ЭТОГО И НЕ АКЦЕНТИРУЕТ - К МИКРОИСТОРИИ И ИСТОРИЧЕСКОЙ АНТРОПОЛОГИИ. ОБРАЩАЮТ НА СЕБЯ ВНИМАНИЕ ОЧЕРКИ О ЯКУНЕ МИРОСЛАВИЧЕ, В КОТОРОМ, КАК В КРИСТАЛЛЕ, ОТРАЖАЕТСЯ СЛОЖНАЯ КЛАНОВО-ТЕРРИТОРИАЛЬНАЯ СТРУКТУРА НОВГОРОДСКОЙ ЭЛИТЫ, ИЛИ О ПЕТРОКЕ И МАРЕНЕ, КОТОРЫЙ, КАК ПИШЕТ АВТОР, ПРИОТКРЫВАЕТ МАЛЕНЬКОЕ ОКНО», ПОЗВОЛЯЮЩЕЕ УВИДЕТЬ ОСОБЕННОСТИ ПОВСЕДНЕВНОЙ ЖИЗНИ СЕМЬИ ИЗ НОВГОРОДСКОЙ ЭЛИТЫ (С. 147), А ТАКЖЕ О МНОГИХ ПЕРСОНАЖАХ БЕРЕСТЯНЫХ ГРАМОТ. В ПРИЛОЖЕНИИ ДАЮТСЯ ПЕРЕВОДЫ НА НЕМЕЦКИЙ ЯЗЫК ВАЖНЕЙШИХ ИСТОЧНИКОВ ПО ИСТОРИИ НОВГОРОДА ИЛИ ОТРЫВКОВ ИЗ НИХ, ЧТО ИМЕЕТ ЦЕННОСТЬ НЕ ТОЛЬКО ДЛЯ НЕМЕЦКОГО ЧИТАТЕЛЯ, ПОСКОЛЬКУ ПЕРЕВОД НА СОВРЕМЕННЫЙ ЯЗЫК ДРЕВНЕГО ИЛИ СРЕДНЕВЕКОВОГО ТЕКСТА - ЭТО ВСЕГДА ИНТЕРПРЕТАЦИЯ.

СРАЗУ ХОТЕЛОСЬ БЫ ПОДЧЕРКНУТЬ, ЧТО, НА МОЙ ВЗГЛЯД, НА СЕГОДНЯШНИЙ ДЕНЬ ЭТО ЛУЧШИЙ В МИРОВОЙ ИСТОРИОГРАФИИ (ВКЛЮЧАЯ И РОССИЙСКУЮ) ОБЩИЙ ОБЗОР ИСТОРИИ НОВГОРОДА И ЕЕ ОСНОВНЫХ ПРОБЛЕМ, И ВСЕ СПЕЦИАЛИСТЫ ТЕПЕРЬ БУДУТ ПРОСТО ОБЯЗАНЫ ОБРАЩАТЬСЯ К МОНОГРАФИИ К. ГЁРКЕ. В КАЧЕСТВЕ ЕЁ «КОНКУРЕНТОВ» МОГУТ РАССМАТРИВАТЬСЯ ДВЕ ПОЯВИВШИЕСЯ ОТНОСИТЕЛЬНО НЕДАВНО КНИГИ: ОПУБЛИКОВАННЫЕ В 2008 Г. ОЧЕРКИ ПО ИСТОРИИ НОВГОРОДА ВЫДАЮЩЕГОСЯ СОВЕТСКОГО И РОССИЙСКОГО ИСТОРИКА В.Л. ЯНИНА (НЫНЕ, УЖЕ, К СОЖАЛЕНИЮ, ПОКОЙНОГО) И КОЛЛЕКТИВНЫЙ ТРУД, ВЫШЕДШИЙ В 2015 Г. ВО ФРАНЦИИ ПОД РЕДАКЦИЕЙ Ф. ФРИЗОНА И О. СЕВАСТЬЯНОВОЙ ${ }^{2}$ КНИГА В.Л. ЯНИНА, БЕЗУСЛОВНО, ПРЕДСТАВЛЯЕТ БОЛЬШОЙ ИНТЕРЕС, ОБОБЩАЯ СДЕЛАННЫЕ ИМ В ДРУГИХ РАБОТАХ ВЫВОДЫ, НО ЭТО ИМЕННО ЕГО ВЗГЛЯД НА НОВГОРОД И ИМЕННО НА ТЕ АСПЕКТЫ ЕГО ИСТОРИИ, КОТОРЫЕ ИНТЕРЕСОВАЛИ УЧЕНОГО (МНОГИЕ ДРУГИЕ, В ТОМ ЧИСЛЕ ВАЖНЕЙШИЕ, ТАМ ПРОСТО ИГНОРИРУЮТСЯ, НАПРИМЕР ПОЧТИ ПОЛНОСТЬЮ ОТСУТСТВУЕТ ХАРАКТЕРИСТИКА СОЦИАЛЬНОЙ СТРУКТУРЫ НОВГОРОДСКОГО ОБЩЕСТВА). ФРАНЦУЗСКОЕ ИЗДАНИЕ АВТОРУ ЭТИХ СТРОК УЖЕ ПРИХОДИЛОСЬ РЕЦЕНЗИРОВАТЬ, И ЗДЕСЬ ЛИШЬ МОЖНО ПОВТОРИТЬ СЛЕДУЮЩЕЕ: ОНО ОЧЕНЬ НЕРОВНО, А КАЧЕСТВЕННО НАПИСАННЫЕ РАЗДЕЛЫ С ЦЕННЫМИ НАБЛЮДЕНИЯМИ СОСЕДСТВУЮТ СО СЛАБО АРГУМЕНТИРОВАННЫМИ И ДАЖЕ ВЕСЬМА СОМНИТЕЛЬНЫМИ ТЕЗИСАМИ (ОСОБЕННО ЭТО КАСАЕТСЯ ЦЕНТРАЛЬНЫХ ДЛЯ КНИГИ РАЗДЕЛОВ 
О.СЕВАСТЬЯНОВОЙ). МОНОГРАФИЯ К. ГЁРКЕ ПРЕКРАСНО СБАЛАНСИРОВАНА. С ОДНОЙ СТОРОНЫ, В НЕЙ ВПОЛНЕ ЧУВСТВУЕТСЯ АВТОРСКИЙ ПОДХОД И ОБОСНОВЫВАЮТСЯ ОРИГИНАЛЬНЫЕ КОНЦЕПЦИИ, А С ДРУГОЙ СТОРОНЫ, АРГУМЕНТЫ В ПОЛЬЗУ СВОИХ МНЕНИЙ К. ГЁРКЕ СТАРАЕТСЯ ВЗВЕШИВАТЬ НА ТЕХ ЖЕ ВЕСАХ, ЧТО И АРГУМЕНТЫ ТЕХ АВТОРОВ, С КОТОРЫМИ ОН ПОЛЕМИЗИРУЕТ. ВО МНОГОМ ЭТО ОБЕСПЕЧИВАЕТСЯ ОЧЕНЬ ХОРОШИМ ЗНАНИЕМ ИСТОЧНИКОВ И ИСТОРИОГРАФИИ, КОТОРЫЕ В СЖАТОМ, НО В ТО ЖЕ ВРЕМЯ В ДОСТАТОЧНО ПОЛНОМ, ВИДЕ ОХАРАКТЕРИЗОВАНЫ В СООТВЕТСТВУЮЩЕМ РАЗДЕЛЕ (МОЖНО НАЗВАТЬ ЛИШЬ ОТДЕЛЬНЫЕ УПУЩЕНИЯ - НАПРИМЕР, ВАЖНЫЕ ГАНЗЕЙСКИЕ ДОКУМЕНТЫ ПО ИСТОРИИ НОВГОРОДА ОПУБЛИКОВАНЫ НЕ ТОЛЬКО В Liv-, Est- und Curländisches И Hansisches Urkundenbuch, НО И В КОЛЛЕКЦИИ МАТЕРИАЛОВ СЪЕЗДОВ ГАНЗЕЙСКИХ ГОРОДОВ, Hanserecesse). АКТИВНО И УДАЧНО АВТОР ИСПОЛЬЗУЕТ ТАКОЙ ЯРКИЙ, ПОСТОЯННО ПОПОЛНЯЮЩИЙСЯ, НО НЕПРОСТОЙ ТИП ИСТОЧНИКОВ, КАК БЕРЕСТЯНЫЕ ГРАМОТЫ. ЭТО СУЩЕСТВЕННО ПОВЫШАЕТ СТЕПЕНЬ ДОСТОВЕРНОСТИ ВЫВОДОВ. ХОЧЕТСЯ ТАКЖЕ ПОЛНОСТЬЮ ПОДДЕРЖАТЬ АВТОРА В ЕГО КРИТИЧЕСКОМ ОТНОШЕНИИ К РЯДУ КРАСИВЫХ, БРОСКИХ, «НОВАТОРСКИХ», НО БЕЗДОКАЗАТЕЛЬНЫХ, НЕ ОСНОВАННЫХ НА ТЩАТЕЛЬНОМ И НЕПРЕДВЗЯТОМ ИЗУЧЕНИИ ВСЕГО КОМПЛЕКСА ИСТОЧНИКОВ ТЕОРИЙ, ВЫДВИНУТЫХ НОВГОРОДОВЕДАМИ, В ТОМ ЧИСЛЕ И ВЕСЬМА ИМЕНИТЫМИ (С. 90, 122-123, 144, 269-270 и ДР.).

САМА ЦЕЛЬ, КОТОРУЮ СТАВИТ К. ГЁРКЕ, ПРЕДПОЛАГАЕТ ОБЗОРНЫЙ ХАРАКТЕР ТЕКСТА И ОПОРУ В ЗНАЧИТЕЛЬНОЙ СТЕПЕНИ НА АКТУАЛЬНУЮ ИСТОРИОГРАФИЮ. ТЕМ НЕ МЕНЕЕ, В КНИГЕ ПРЕДСТАВЛЕНЫ И РЕЗУЛЬТАТЫ СОБСТВЕННЫХ ИССЛЕДОВАНИЙ АВТОРА, И ЛЮБОПЫТНЫЕ КОНКРЕТНЫЕ НАБЛЮДЕНИЯ. К НЕКОТОРЫМ ИЗ НИХ ХОТЕЛОСЬ БЫ ПРИВЛЕЧЬ ОСОБОЕ ВНИМАНИЕ ЧИТАТЕЛЕЙ.

ЗАСЛУЖИВАЕТ ИНТЕРЕСА ОСТРОУМНАЯ ТРАКТОВКА АВТОРОМ ЗАГАДОЧНОГО НАИМЕНОВАНИЯ НОВГОРОДА. ПРЕДЛАГАЛИСЬ, КАК ИЗВЕСТНО, РАЗНЫЕ ВЕРСИИ ПО ПОВОДУ ТОГО, ПО ОТНОШЕНИЮ К ЧЕМУ ГОРОД НА ВОЛХОВЕ БЫЛ «НОВЫМ». ПО МНЕНИЮ К. ГЁРКЕ (С. 60-61), ПЕРВОНАЧАЛЬНЫЙ СМЫСЛ НАИМЕНОВАНИЯ «НОВГОРОД» НЕ «НОВЫЙ ГОРОД», А «НОВАЯ КРЕПОСТЬ» (НОВАЯ ПО ОТНОШЕНИЮ К «СТАРОЙ» КРЕПОСТИ, ЗАЛОЖЕННОЙ ПРЕДПОЛОЖИТЕЛЬНО РЮРИКОМ). И ТОЛЬКО ПОЗДНЕЕ «НОВГОРОД» БЫЛ ПЕРЕОСМЫСЛЕН КАК ГОРОД. К САМЫМ СИЛЬНЫМ СТОРОНАМ КНИГИ ОТНОСИТСЯ ПОДРОБНАЯ И УБЕДИТЕЛЬНАЯ ТРАКТОВКА СОЦИАЛЬНОГО СТРОЯ И ДЕМОГРАФИЧЕСКОЙ СИТУАЦИИ НОВГОРОДА - СЮЖЕТАМ, КОТОРЫЕ ЛИБО ПОЧТИ ПОЛНОСТЬЮ ИГНОРИРУЮТСЯ В ИСТОРИОГРАФИИ, ЛИБО ПОЛУЧИЛИ ЛИШЬ ФРАГМЕНТАРНУЮ И ЗАЧАСТУЮ ВЕСЬМА СПОРНУЮ ИНТЕРПРЕТАЦИЮ. ЭТО, РАЗУМЕЕТСЯ, НЕ СЛУЧАЙНО: РЕЧЬ ИДЁТ О РЕЗУЛЬТАТАХ СОБСТВЕННЫХ ИЗЫСКАНИЙ К. ГЁРКЕ. ИЗ НОВОГО - ЗАСЛУЖИВАЕТ ВНИМАНИЕ ПРЕДПОЛОЖЕНИЕ АВТОРА О ТОМ, ЧТО К ЧЁРНЫМ ЛЮДЯМ В НОВГОРОДЕ XIV-XV ВВ. ПРИНАДЛЕЖАЛИ НЕ ТОЛЬКО МЕЛКИЕ ТОРГОВЦЫ И РЕМЕСЛЕННИКИ, КАК ЭТО ОБЫЧНО ПРЕДПОЛАГАЕТСЯ, НО И ДОСТАТОЧНО МНОГОЧИСЛЕННЫЕ - СУДЯ ПО ГАНЗЕЙСКИМ ДОКУМЕНТАМ - ЛОДЕЙЩИКИ И НОСИЛЬЩИКИ, Т.Е. НАЁМНЫЕ РАБОТНИКИ, ЗАНИМАВШИЕСЯ ТРАНСПОРТИРОВКОЙ КУПЕЧЕСКИХ ТОВАРОВ.

ПОДРОБНО И С ПРИВЛЕЧЕНИЕМ ОСНОВНЫХ ИСТОЧНИКОВ АВТОР РАССКАЗЫВАЕТ О ПРИСОЕДИНЕНИИ НОВГОРОДА К МОСКВЕ. ИНТЕРЕСНА ЕГО ТРАКТОВКА СОГЛАШЕНИЯ 1471 Г. НОВГОРОДА С ВЕЛИКИМ КНЯЖЕСТВОМ ЛИТОВСКИМ: ИСТОРИК СКЛОНЯЕТСЯ К УЖЕ ВЫСКАЗЫВАВШЕМУСЯ В ЛИТЕРАТУРЕ МНЕНИЮ О ТОМ, ЧТО ЭТО БЫЛ ПРОЕКТ 
ДОГОВОРА, ПРИЧЁМ ПРОЕКТ НОВГОРОДСКИЙ, СОСТАВЛЕННЫЙ СПЕШНО, БУКВАЛЬНО НАКАНУНЕ БИТВЫ НА ШЕЛОНИ .

СКАЗАННОЕ ВЫШЕ НЕ ОЗНАЧАЕТ, ЧТО АБСОЛЮТНО СО ВСЕМ В БОЛЬШОЙ КНИГЕ К. ГЁРКЕ МОЖНО СОГЛАСИТЬСЯ. СТРУКТУРА ИЗЛОЖЕНИЯ ПОСТРОЕНА В ЦЕЛОМ ПО ХРОНОЛОГИЧЕСКОМУ ПРИНЦИПУ. ЭТО, БЕССПОРНО, ПРОСТО И УДОБНО, НО НЕКОТОРЫЕ «СКВОЗНЫЕ» ТЕМЫ ЛУЧШЕ БЫЛО БЫ ДЛЯ УДОБСТВА ЧИТАТЕЛЕЙ, КАЖЕТСЯ, ОСВЕТИТЬ ПОСЛЕДОВАТЕЛЬНО В ОДНОМ МЕСТЕ, ТЕМ БОЛЕЕ ЧТО ВСЁ РАВНО СОХРАНИТЬ ХРОНОЛОГИЧЕСКИЙ РИГОРИЗМ НЕВОЗМОЖНО.

ЕСТЬ И ОПРЕДЕЛЁННЫЕ УПУЩЕНИЯ БОЛЕЕ ЧАСТНОГО ХАРАКТЕРА (ЧТО, НА ВЗГЛЯД РЕЦЕНЗЕНТА, ЕСТЕСТВЕННО). ГОВОРЯ О ПРОИСХОЖДЕНИИ НОВГОРОДА, МОЖНО БЫЛО БЫ ОСТАНОВИТЬСЯ НА ИНТЕРЕСНОЙ ГИПОТЕЗЕ Б.Н.ФЛОРИ О ДВУХ РАННИХ ПОЛИТИЧЕСКИХ ЦЕНТРАХ - КНЯЖЕСКОМ (И СКАНДИНАВСКОМ) ГОРОДИЩЕ И БУДУЩЕМ НОВГОРОДЕ КАК МЕСТЕ ПЛЕМЕННЫХ СОБРАНИЙ ИЛЬМЕНСКИХ СЛОВЕН ${ }^{4}$. СУЩЕСТВЕННО БО́ЛЬШИМ УПУЩЕНИЕМ СЛЕДУЕТ ПРИЗНАТЬ ИГНОРИРОВАНИЕ ВАЖНЕЙШИХ РАБОТ А.А. ГИППИУСА, В КОТОРЫХ ПРОВЕДЕНА СТРАТИФИКАЦИЯ НОВГОРОДСКОЙ ВЛАДЫЧНОЙ ЛЕТОПИСИ И ОПРЕДЕЛЕНА ПРИНАДЛЕЖНОСТЬ РАЗЛИЧНЫХ СЕГМЕНТОВ ЕЁ ТЕКСТА ТЕМ ИЛИ ИНЫМ ЛЕТОПИСЦАМ, РАБОТАВШИМ ПРИ РАЗНЫХ НОВГОРОДСКИХ АРХИЕРЕЯХ 5 . «ВЕСЬ НОВГОРОД» - ЭТО НИ В КОЕЙ МЕРЕ НЕ НАЗВАНИЕ НОВГОРОДСКОГО ГОСУДАРСТВА (des Staates) (c. 81), А ОБОЗНАЧЕНИЕ НОВГОРОДСКОГО ПОЛИТИЧЕСКОГО СООБЩЕСТВА, КОТОРОЕ АВТОР ЭТИХ СТРОК ПРЕДЛАГАЕТ НАЗЫВАТЬ «ПОЛИТИЧЕСКИМ НАРОДОМ». ЕСЛИ И МОЖНО ПРИМЕНИТЕЛЬНО К СРЕДНЕВЕКОВОМУ НОВГОРОДУ ГОВОРИТЬ ОБ АБСТРАКТНОМ ГОСУДАРСТВЕ, ТО ОНО НАЗЫВАЛОСЬ СНАЧАЛА ПРОСТО «НОВГОРОД», А ПОЗДНЕЕ - С XIV В. - «ВЕЛИКИЙ НОВГОРОД». ПРАВИЛЬНЕЕ ГОВОРИТЬ НЕ О «ЛИВОНСКОМ ОРДЕНЕ», А О ЛИВОНСКОМ ОТДЕЛЕНИИ (ЛАНДМЕЙСТЕРСТВЕ) ТЕВТОНСКОГО ОРДЕНА (ИЛИ О ТЕВТОНСКОМ ОРДЕНЕ В ЛИВОНИИ). В ПОСЛЕДНИЕ ДЕСЯТИЛЕТИЯ ПОЯВИЛИСЬ НОВЕЙШИЕ ИССЛЕДОВАНИЯ НОВГОРОДСКОГО СЕЛЬСКОГО ХОЗЯЙСТВА (В ОСНОВНОМ БРИТАНСКИХ УЧЁНЫХ), В КОТОРЫХ ТЩАТЕЛЬНО АНАЛИЗИРУЕТСЯ БОГАТЫЙ АРХЕОЛОГИЧЕСКИЙ МАТЕРИАЛ 6 . В РЕЗУЛЬТАТЕ КАРТИНА НОВГОРОДСКОЙ ЭКОНОМИКИ ОКАЗЫВАЕТСЯ ГОРАЗДО БОЛЕЕ СЛОЖНОЙ, ЧЕМ ПРЕДСТАВЛЯЛОСЬ РАНЕЕ. К. ГЁРКЕ УДЕЛЯЕТ ЗАСЛУЖЕННО БОЛЬШОЕ МЕСТО НОВГОРОДСКОЙ ТОРГОВЛЕ И ОЧЕНЬ ПОДРОБНО ХАРАКТЕРИЗУЕТ ДАЖЕ ЕЁ РАННИЙ, ДОГАНЗЕЙСКИЙ, ПЕРИОД, НЕ ГОВОРЯ УЖЕ О ТОРГОВЛЕ С ГАНЗОЙ, КОТОРОЙ ПОСВЯЩЁН БОЛЬШОЙ РАЗДЕЛ, НАПИСАННЫЙ НА ОСНОВЕ БОГАТЫХ СВЕДЕНИЙ ГАНЗЕЙСКИХ ДОКУМЕНТОВ. ПРИ ЭТОМ АВТОР ПОЧЕМУ-ТО ПОВТОРЯЕТ СТАРЫЙ МИФ ОБ ЭКСПОРТЕ МЁДА ИЗ НОВГОРОДА (c. 91, 304), ОПРОВЕРГНУТЫЙ ЕЩЁ Л.К. ГЁТЦЕМ И А.Л. ХОРОШКЕВИЧ ${ }^{7}$.

9 ПРИ ВСЕЙ СПРАВЕДЛИВОСТИ КРИТИЧЕСКОГО ОТНОШЕНИЯ АВТОРА К ПОЗДНИМ ИСТОЧНИКАМ И СЛАБО АРГУМЕНТИРОВАННЫМ ПОСТРОЕНИЯМ ИСТОРИКОВ НЕСКОЛЬКО СТРАННЫМ ВЫГЛЯДИТ ЕГО ДОВЕРИЕ К РАССКАЗУ ТАК НАЗЫВАЕМОЙ ИОАКИМОВСКОЙ ЛЕТОПИСИ О КРЕЩЕНИИ НОВГОРОДА. ЗДЕСЬ ПОЛЕЗНО БЫЛО БЫ ОБРАТИТЬСЯ К КНИГЕ А.П. ТОЛОЧКО, В КОТОРОЙ РАССМАТРИВАЕТСЯ ЭТОТ ВОПРОС ${ }^{8}$. КАК ПРЕДСТАВЛЯЕТСЯ, АВТОР ПРЕУВЕЛИЧИВАЕТ СТЕПЕНЬ УСТОЙЧИВОСТИ ЯЗЫЧЕСКИХ ПЕРЕЖИТКОВ В НОВГОРОДЕ ХІІ-ХІІІ ВВ. И НАПРАСНО (ВСЛЕД ЗА И.Я. ФРОЯНОВЫМ) СВЯЗЫВАЕТ КАЗНЬ В 1227 Г. ВОЛХВОВ С ВЫСТУПЛЕНИЕМ НОВГОРОДЦЕВ ПРОТИВ АРХИЕПИСКОПА, ДЛЯ ЧЕГО ЛЕТОПИСЬ НЕ ДАЁТ НИКАКИХ ОСНОВАНИЙ. СПОРНЫМ ВЫГЛЯДИТ ВЫБОР ПОНЯТИЯ «ШАМАН» ДЛЯ ПЕРЕДАЧИ ДРЕВНЕРУССКОГО «ВОЛХВ»: ОБЫЧНО СЧИТАЕТСЯ, ЧТО ТАК 
МОГЛИ НАЗЫВАТЬ ЯЗЫЧЕСКИХ ЖРЕЦОВ ИЛИ КОЛДУНОВ. ОСНОВЫВАЯСЬ НА ЭТИМОЛОГИИ СРЕДНЕНИЖНЕНЕМЕЦКОЙ ПЕРЕДАЧИ НОВГОРОДСКОГО ТЫСЯЦКОГО, hertoge (hertoch), К. ГЁРКЕ СЧИТАЕТ, ЧТО ИЗНАЧАЛЬНОЙ ФУНКЦИЕЙ ТЫСЯЦКОГО БЫЛА ВОЕННАЯ. ЭТИМОЛОГИЯ СРЕДНЕНИЖНЕНЕМЕЦКОГО СЛОВА ДЕЙСТВИТЕЛЬНО ВПОЛНЕ ПРОЗРАЧНАЯ (ВОЕНАЧАЛЬНИК); НА ЭТО ЖЕ ВРОДЕ БЫ НАМЕКАЕТ И ПРОИСХОЖДЕНИЕ РУССКОГО ОБОЗНАЧЕНИЯ «ТЫСЯЦКИЙ» (СР. «ВОЕВ СЛАВНЫХ ТЫСЯЩУ» В ЛЕТОПИСНОМ РАССКАЗЕ О КОНФЛИКТЕ В НОВГОРОДЕ В $\left.1015 \Gamma^{9}{ }^{9}\right)$. ОДНАКО ПО ИСТОЧНИКАМ, В КОТОРЫХ УПОМИНАЮТСЯ ТЫСЯЦКИЕ, ЭТОГО НЕ ВИДНО, А ИЗ ГАНЗЕЙСКИХ ДОКУМЕНТОВ XIII-XV ВВ. ЯСНО СЛЕДУЕТ, ЧТО ТЫСЯЦКИЙ ВЕДАЛ ПРЕЖДЕ ВСЕГО СУДОМ ПО ТОРГОВЫМ ДЕЛАМ. ВОПРОС ПОКА ПРИХОДИТСЯ ОСТАВИТЬ ОТКРЫТЫМ. К СОЖАЛЕНИЮ, В РАБОТЕ К. ГЁРКЕ НЕ УЧТЕНА СТАТЬЯ В.А. КУЧКИНА О НОВГОРОДСКИХ ТЫСЯЦКИХ (КАК И ЕГО ТРУДЫ О ДРЕВНЕРУССКОЙ ДЕЦИМАЛЬНОЙ ОРГАНИЗАЦИИ ВООБЩЕ), ГДЕ ПРЕДЛАГАЕТСЯ ТРАКТОВКА ТЫСЯЦКИХ КАК ДОЛЖНОСТНЫХ ЛИЦ (СНАЧАЛА КНЯЖЕСКИХ, ПОТОМ - РЕСПУБЛИКАНСКИХ), ОТВЕТСТВЕННЫХ ПРЕЖДЕ ВСЕГО ЗА ХОЗЯЙСТВЕННУЮ СФЕРУ ${ }^{10}$. НАПРАСНО АВТОР СОГЛАШАЕТСЯ С ЯВНО ПРЕУВЕЛИЧЕННЫМИ ОЦЕНКАМИ ЗНАЧЕНИЯ КНЯЗЯ В ДОМОНГОЛЬСКОМ НОВГОРОДЕ, КОТОРЫЕ ДАЁТ В СВОЕЙ СТАТЬЕ М.К. ПОЛ (ТАК, У КНЯЗЯ, КОНЕЧНО ЖЕ, НЕ БЫЛО ЭКСКЛЮЗИВНОГО ПРАВА НА СОЗЫВ ВЕЧА) (С. 125). К. Г̈̈РЕ ВПОЛНЕ СПРАВЕДЛИВО ОТМЕЧАЕТ, ЧТО ВЕЧЕВОЕ ИЗБРАНИЕ АРХИЕПИСКОПОВ В НОВГОРОДЕ «НЕ УПАЛО С НЕБА» И ВПОЛНЕ АДЕКВАТНО ХАРАКТЕРИЗУЕТ ЦЕРКОВНО-ПОЛИТИЧЕСКИЙ КОНТЕКСТ ЕГО ВОЗНИКНОВЕНИЯ (С. 129-132), НО НИЧЕГО НЕ ПИШЕТ О ПРОИСХОЖДЕНИИ САМОЙ ФОРМЫ ИЗБРАНИЯ (ПО ЖРЕБИЮ ИЗ ТРЁХ КАНДИДАТОВ НА ВЕЧЕ), МЕЖДУ ТЕМ ЭТО ВЕСЬМА ИНТЕРЕСНЫЙ И ДИСКУССИОННЫЙ ВОПРОС. КОНЦЕПЦИЯ А.В.ПЕТРОВА ОТНОСИТЕЛЬНО ОСНОВОПОЛАГАЮЩЕЙ РОЛИ «СТОРОН» В СОЦИАЛЬНО-ПОЛИТИЧЕСКОЙ БОРЬБЕ В ДОМОНГОЛЬСКОМ НОВГОРОДЕ И, ТЕМ БОЛЕЕ, ЕГО ИДЕЯ ОБ ИХ АРХАИЧЕСКОМ «ФРАТРИАЛЬНОМ» ХАРАКТЕРЕ УЖЕ ПОДВЕРГАЛИСЬ КРИТИКЕ ${ }^{11}$. ВАЖНО И ТО, ЧТО, В ОТЛИЧИЕ ОТ АНТИЧНЫХ ФРАТРИЙ, СТОРОНЫ - В ОТЛИЧИЕ ОТ «КОНЦОВ» - БЫЛИ НЕФОРМАЛЬНЫМИ ОБЪЕДИНЕНИЯМИ: НЕ СУЩЕСТВОВАЛО НИКАКИХ ПОСТОЯННЫХ ПОЛИТИЧЕСКИХ ИНСТИТУТОВ, ПРЕДСТАВЛЯВШИХ СОФИЙСКУЮ И ТОРГОВУЮ СТОРОНЫ. В ДЛИТЕЛЬНОЙ ДИСКУССИИ ПО ПОВОДУ ЗНАЧЕНИЯ ФОРМУЛЫ «ОГНИЩАНЕ-ГРИДЬКУПЦЫ» К. ГЁРКЕ ПРИСОЕДИНЯЕТСЯ К СТОРОННИКАМ ЕЁ «УЗКОЙ» ТРАКТОВКИ (ОГНИЩАНЕ - КНЯЖЕСКИЕ ДРУЖИННИКИ, ГРИДЬ - ПРОФЕССИОНАЛЬНЫЕ ВОИНЫ, КУПЦЫ - ТОРГОВЦЫ). ЭТО, НЕСОМНЕННО, ОЧЕНЬ СЛОЖНЫЙ ВОПРОС. ОДНАКО В НАСТОЯЩЕЕ ВРЕМЯ Я СКЛОНЯЮСЬ К ДРУГОЙ ТРАКТОВКЕ. В РАМКАХ УЗКОЙ» ИНТЕРПРЕТАЦИИ НЕВОЗМОЖНО ОБЪЯСНИТЬ, ЗАЧЕМ КНЯЗЬЯМ НАДО БЫЛО ВЕСТИ ПЕРЕГОВОРЫ СО СВОИМИ ЖЕ СЛУЖИЛЫМИ ЛЮДЬМИ, А НОВЫЕ АРГУМЕНТЫ, КОТОРЫЕ ПРИВОДИТ ИСТОРИК, НЕ ВЫГЛЯДЯТ УБЕДИТЕЛЬНЫМИ. ТАК, К. ГЁРКЕ В ДОКАЗАТЕЛЬСТВО САМОГО СУЩЕСТВОВАНИЯ СЛОЯ ПРОФЕССИОНАЛЬНЫХ ВОИНОВ В НОВГОРОДЕ ССЫЛАЕТСЯ НА УПОМИНАНИЯ «ДРУЖИН» У НОВГОРОДСКИХ БОЯР. НО ВО ВСЕХ СЛУЧАЯХ, КОТОРЫЕ ОН ПРИВОДИТ, «ДРУЖИНА» - ЭТО НЕ ПРОФЕССИОНАЛЬНЫЕ ВОИНЫ, А «ДРУЗЬЯ», ПРИБЛИЖЁННЫЕ, В САМОМ ОБЩЕМ СМЫСЛЕ СЛОВА. ВО ВСЯКОМ СЛУЧАЕ, ТАКОМУ ПОНИМАНИЮ НИЧТО НЕ ПРОТИВОРЕЧИТ. НАКОНЕЦ, РЕШАЮЩЕЕ ДОКАЗАТЕЛЬСТВО В ПОЛЬЗУ «ШИРОКОГО» ПОНИМАНИЯ ФОРМУЛЫ (ОГНИЩАНЕ И ГРИДЬ - «ВЯЧШИЕ», ВОЗМОЖНО БОЯРЕ И БУДУЩИЕ ЖИТЬИ; «КУПЦЫ», «МЕНЬШИЕ», Т.Е. ТОРГОВЦЫ И РЕМЕСЛЕННИКИ) - ТОТ ФАКТ, ЧТО В НОВГОРОДСКОМ (И ДРЕВНЕРУССКОМ) СОЦИАЛЬНОПОЛИТИЧЕСКОМ ЯЗЫКЕ НЕ БЫЛО ОБОЗНАЧЕНИЯ РЕМЕСЛЕННИКОВ ВО МНОЖЕСТВЕННОМ ЧИСЛЕ, ХОТЯ РЕМЕСЛЕННИКИ, БЕЗУСЛОВНО, В НОВГОРОДЕ БЫЛИ И ИГРАЛИ 
ОПРЕДЕЛЁННУЮ ПОЛИТИЧЕСКУЮ РОЛЬ, А ПРЕДСТАВИТЕЛИ ОТДЕЛЬНЫХ РЕМЕСЛЕННЫХ СПЕЦИАЛЬНОСТЕЙ НЕОДНОКРАТНО УПОМИНАЮТСЯ В ИСТОЧНИКАХ. НЕТ БОЛЬШИХ СОМНЕНИЙ В ТОМ, ЧТО ПОД «КУПЦАМИ» ПОДРАЗУМЕВАЛИ И ИХ, ТЕМ БОЛЕЕ, ЧТО В НОВГОРОДЕ МЕЛКАЯ ТОРГОВЛЯ И РЕМЕСЛО НЕ БЫЛИ ОТДЕЛЕНЫ ДРУГ ОТ ДРУГА. А ЭТО, В СВОЮ ОЧЕРЕДЬ, ОЗНАЧАЕТ, ЧТО И ДРУГИЕ ЧАСТИ ЭТОЙ ФОРМУЛЫ ДОЛЖНЫ ОБОЗНАЧАТЬ ВСЮ НОВГОРОДСКУЮ ЭЛИТУ В ЦЕЛОМ, А НЕ КАКИЕ-ТО ЕЁ СПЕЦИФИЧЕСКИЕ ГРУППЫ. В ЦЕЛОМ, КАК ПРЕДСТАВЛЯЕТСЯ, В ФОРМУЛЕ «ОГНИЩАНЕ-ГРИДЬ-КУПЦЫ» НАДО ВИДЕТЬ НЕ ПОЛУЧИВШИЙ РАЗВИТИЯ РАННИЙ ВАРИАНТ ОБОЗНАЧЕНИЯ НОВГОРОДСКОГО «ПОЛИТИЧЕСКОГО НАРОДА», МЕСТО КОТОРОГО ЗАНЯЛО В КОНЦЕ XII В. БОЛЕЕ ЯСНОЕ И ЛАКОНИЧНОЕ ВЫРАЖЕНИЕ «ВЕСЬ НОВГОРОД». ОТНОСИТЕЛЬНО ОГНИЩАН И ГРИДЕЙ УДАЧНОЕ ОБЪЯСНЕНИЕ БЫЛО ПРЕДЛОЖЕНО УЖЕ ДОВОЛЬНО ДАВНО А.А.ГОРСКИМ: В ЭТИХ АРХАИЧЕСКИХ ОБОЗНАЧЕНИЯХ СЛЕДУЕТ ВИДЕТЬ СЛЕДЫ ДРУЖИННОГО ПРОИСХОЖДЕНИЯ ЗНАЧИТЕЛЬНОЙ ЧАСТИ НОВГОРОДСКОЙ ЭЛИТЫ ${ }^{12}$. К. ГЁРКЕ ССЫЛАЕТСЯ НА СТАТЬЮ М.Х. АЛЕШКОВСКОГО, НО У ТОГО ИДЁТ РЕЧЬ КАК РАЗ О ТОМ, ЧТО В ХІІ-ХІІІ ВВ. КУПЦАМИ МОГЛИ НАЗЫВАТЬ ВСЁ ТОРГОВО-РЕМЕСЛЕННОЕ НАСЕЛЕНИЕ НОВГОРОДА, А ЭТО ПРЯМО ПРОТИВОРЕЧИТ «УЗКОЙ» ИНТЕРПРЕТАЦИИ ${ }^{13}$. ВЕСЬМА СОМНИТЕЛЬНЫМ КАЖЕТСЯ ОТНЕСЕНИЕ ДУХОВЕНСТВА В ЦЕЛОМ В ХІІ В. К «МЕНЬШИМ» И К НИМ ЖЕ - «КРЕСТЬЯН» (Bauern), С КОТОРЫМИ АВТОР ОТОЖДЕСТВЛЯЕТ СМЕРДОВ. ПРЕДСТАВЛЯЕТСЯ, ЧТО СРЕДИ ДУХОВЕНСТВА БЫЛИ КАК ВЯЧШИЕ, ТАК И МЕНЬШИЕ, А СМЕРДЫ ВООБЩЕ НЕ ВХОДИЛИ В СОСТАВ НОВГОРОДСКОГО ПОЛИТИЧЕСКОГО СООБЩЕСТВА, ЧАСТЬЮ КОТОРОГО, НЕСОМНЕННО, БЫЛИ МЕНЬШИЕ.

ОЦЕНКА АЛЕКСАНДРА НЕВСКОГО В РАБОТЕ К. ГЁРКЕ В ЦЕЛОМ ВЗВЕШЕННАЯ (С. 189-193), И АВТОР СПРАВЕДЛИВО СЧИТАЕТ ЕГО НЕ НОСИТЕЛЕМ КАКОГО-ТО ИДЕОЛОГИЧЕСКОГО ВНЕШНЕПОЛИТИЧЕСКОГО КУРСА В ДУХЕ ГЕОПОЛИТИКИ ХІХ-ХХ ВВ., ДЛЯ ЧЕГО НЕТ НИКАКИХ ОСНОВАНИЙ, А ПОЛИТИКОМ-ПРАГМАТИКОМ. ОТКАЗ ОТ ПРЯМОГО ПРОТИВОСТОЯНИЯ МОНГОЛО-ТАТАРАМ, ДЕЙСТВИТЕЛЬНО, БЫЛ БЕЗАЛЬТЕРНАТИВНЫМ (ХОТЯ АЛЕКСАНДР НЕВСКИЙ, ВИДИМО, НА КАКОМ-ТО ЭТАПЕ ВПОЛНЕ ВСЕРЬЁЗ РАССМАТРИВАЛ ВОЗМОЖНОСТЬ ЗАКЛЮЧЕНИЯ СОЮЗА С ЗАПАДОМ ПРОТИВ МОНГОЛОВ И ГОТОВ БЫЛ РАДИ ЭТОГО ПОЙТИ НА ОПРЕДЕЛЁННЫЕ УСТУПКИ В ХОДЕ ПЕРЕГОВОРОВ С ПАПОЙ РИМСКИМ). ОДНАКО ВРЯД ЛИ МОЖНО СОГЛАСИТЬСЯ С ТЕМ, ЧТО В НОВГОРОДСКОЙ ПЕРВОЙ ЛЕТОПИСИ СТАРШЕГО ИЗВОДА (ДАЛЕЕ: НПЛ СТ.) «НЕТ НИКАКИХ СЛЕДОВ» ВОСПРИЯТИЯ АЛЕКСАНДРА ЯРОСЛАВИЧА КАК ГЕРОЯ. В СИТУАЦИИ ПРОТИВОСТОЯНИЯ «МЕНЬШИХ» НОВГОРОДЦЕВ С АЛЕКСАНДРОМ В 1255 Г. ВЛАДЫЧНЫЙ ЛЕТОПИСЕЦ ОПРАВДЫВАЕТ КНЯЗЯ («А КНЯЗЬ БЕЗЪ ГРЪХА») И ПЕРЕКЛАДЫВАЕТ ВИНУ НА НОВГОРОДСКИХ «КРЕСТОПРЕСТУПНИКОВ». НЕ ОСУЖДАЕТ ЛЕТОПИСЕЦ КНЯЗЯ И ПРИ ОПИСАНИИ ТАТАРСКОГО ЧИСЛА В 1259 Г., КОГДА АЛЕКСАНДР ПОМОГАЛ ОБЕСПЕЧИВАТЬ ПРОВЕДЕНИЕ ПЕРЕПИСИ В НОВГОРОДЕ. И ИМЕННО В НПЛ СТ. СОДЕРЖИТСЯ ЗНАМЕНИТЫЙ НЕКРОЛОГ АЛЕКСАНДРА С ЯВНО «ГЕРОИЧЕСКИМИ» МОТИВАМИ, НЕ ОСТАВЛЯЮЩИМИ СОМНЕНИЯ В ТОМ, ЧТО, НЕСМОТРЯ НА ВСЕ СЛОЖНОСТИ В ОТНОШЕНИЯХ МЕЖДУ НИМ И НОВГОРОДОМ, ТАМ ВЫСОКО ОЦЕНИВАЛИ ЕГО КАК ЗАСТУПНИКА ВСЕЙ РУССКОЙ ЗЕМЛИ И НОВГОРОДА В ЧАСТНОСТИ: «...ДАИ, ГОСПОДИ МИЛОСТИВЫИ, ВИДЪТИ ЕМУ ЛИЦЕ ТВОЕ В БУДУЩИИ ВЪКЪ, ИЖЕ ПОТРУДИСЯ ЗА НОВЪГОРОДЪ И ЗА ВСЮ РУСЬСКУЮ ЗЕМЛЮ» ${ }^{14}$. КАК УСТАНОВИЛ А.А. ГИППИУС, АВТОРОМ ЭТОЙ ЧАСТИ ЛЕТОПИСИ БЫЛ ТИМОФЕЙ ПОНОМАРЬ, ВЛАДЫЧНЫЙ НОТАРИЙ И ЛЕТОПИСЕЦ НОВГОРОДСКИХ АРХИЕПИСКОПОВ СПИРИДОНА И ДАЛМАТА ${ }^{15}$. ОН БЫЛ УБЕЖДЁННЫМ ЗАщИТНИКОМ НОВГОРОДСКИХ ВОЛЬНОСТЕЙ («ПРАВДЫ 
НОВГОРОДСКОЙ»), И ДАЖЕ ЕМУ БЫЛ НЕ ЧУЖД «ГЕРОИЧЕСКИЙ КУЛЬТ» АЛЕКСАНДРА НЕВСКОГО.

В РАССКАЗЕ О ДЕЯТЕЛЬНОСТИ АРХИЕПИСКОПА ЕВФИМИЯ ІІ СЛЕДОВАЛО БЫ, НАВЕРНОЕ, ПОДЧЕРКНУТЬ ТОТ ФАКТ, ЧТО В НОВГОРОДЕ ФЛОРЕНТИЙСКАЯ УНИЯ БЫЛА ОСУЖДЕНА РАНЬШЕ, ЧЕМ В МОСКВЕ, ЧТО, В ЧАСТНОСТИ, ПОКАЗЫВАЕТ НЕАДЕКВАТНОСТЬ РЕАЛЬНОМУ ПОЛОЖЕНИЮ ДЕЛ ОБВИНЕНИЙ НОВГОРОДЦЕВ СО СТОРОНЫ МОСКОВСКОЙ ПРОПАГАНДЫ В «ИЗМЕНЕ» ПРАВОСЛАВИЮ. ГОВОРЯ О СТРОИТЕЛЬСТВЕ В НОВГОРОДЕ ЭТОГО ВРЕМЕНИ, МОЖНО БЫЛО БЫ ЗАТРОНУТЬ ИЗОБРАЖЕНИЕ СВЯТОЙ СОФИИ В ОБРАЗЕ ОГНЕЗРАЧНОГО АНГЕЛА, ПРЕДСТАВЛЯЮЩЕЕ СОБОЙ, СКОРЕЕ ВСЕГО, ВИЗУАЛИЗАЦИЮ НЕБЕСНОГО ПАТРОНА НОВГОРОДА, КУЛЬТ КОТОРОГО С ХІІІ В. НАХОДИЛСЯ В ЦЕНТРЕ НОВГОРОДСКОЙ ПОЛИТИЧЕСКОЙ ИДЕОЛОГИИ. НОВГОРОДСКОЙ ПОЛИТИЧЕСКОЙ РИТОРИКЕ И МИФОЛОГИИ (ВЕСЬМА БОГАТЫМ, ОТРАЗИВШИМСЯ ВО МНОГИХ ИСТОЧНИКАХ: ЛЕТОПИСАНИЕ, АГИОГРАФИЧЕСКИЕ СОЧИНЕНИЯ, ИКОНЫ И ФРЕСКИ) В КНИГЕ ВООБЩЕ УДЕЛЯЕТСЯ МАЛО ВНИМАНИЯ. МЕЖДУ ТЕМ, ВАЖНОСТЬ ЭТОГО АСПЕКТА ЯВСТВУЕТ ИЗ ТАКОГО ПРИМЕРА: АМБИВАЛЕНТНОЕ САМОВОСПРИЯТИЕ НОВГОРОДА ВТОРОЙ ПОЛОВИНЫ ХІІІ - XV ВВ. КАК ОДНОВРЕМЕННО ВОЛЬНОГО ГОРОДА И «ОТЧИНЫ» ВЕЛИКИХ КНЯЗЕЙ ВЛАДИМИРСКИХ, НЕСОМНЕННО, ОКАЗАЛО ВОЗДЕЙСТВИЕ НА МОРАЛЬНО-ПОЛИТИЧЕСКОЕ СОСТОЯНИЕ НОВГОРОДЦЕВ В РЕШАЮЩИЙ МОМЕНТ ПРОТИВОСТОЯНИЯ С МОСКВОЙ.

В ИНТЕРЕСНОМ РАЗДЕЛЕ, ПОСВЯЩЁННОМ ВОСПРИЯТИЮ НОВГОРОДА В НОВОЕ И НОВЕЙШЕЕ ВРЕМЯ И ЕГО «ИНСТРУМЕНТАЛИЗАЦИИ», АВТОР ПОЧЕМУ-ТО ИГНОРИРУЕТ ЗЕМСКО-ВЕЧЕВУЮ ШКОЛУ И, ПРЕЖДЕ ВСЕГО, РАБОТЫ В.И. СЕРГЕЕВИЧА ${ }^{16}$. МЕЖДУ ТЕМ, ОНИ ОКАЗАЛИ СУЩЕСТВЕННО БОЛЬШЕЕ ВЛИЯНИЕ НА НАУЧНЫЕ ПРЕДСТАВЛЕНИЯ О СОЦИАЛЬНО-ПОЛИТИЧЕСКОМ СТРОЕ НОВГОРОДА, ЧЕМ БОЛЕЕ ПОПУЛЯРНЫЕ, НО СУЩЕСТВЕННО МЕНЕЕ ФУНДИРОВАННЫЕ СОЧИНЕНИЯ Н.И. КОСТОМАРОВА, О КОТОРЫХ ДОВОЛЬНО ПОДРОБНО ПИШЕТ К. ГЁРКЕ (С. 398-399). В ЧАСТНОСТИ, ИМЕННО НА ЗЕМСКОВЕЧЕВОЙ ТЕОРИИ (НАРЯДУ СО СПЕЦИФИЧЕСКОЙ ИНТЕРПРЕТАЦИЕЙ МАРКСИЗМА) БАЗИРОВАЛАСЬ «ШКОЛА И.Я. ФРОЯНОВА». ЕСЛИ ГОВОРИТЬ О ПОСЛЕДНЕЙ, ТО ОНА ДЕЙСТВИТЕЛЬНО ЯВЛЯЕТ СОБОЙ «ИНСТРУМЕНТАЛИЗАЦИИ» КАК НОВГОРОДА, ТАК И ДРУГИХ ТЕМ ИЗ РУССКОЙ ИСТОРИИ, НО АВТОР ПРЕДСТАВЛЯЕТ ЭТО СЕБЕ ДОВОЛЬНО СХЕМАТИЧНО. В ДЕЙСТВИТЕЛЬНОСТИ, ЦЕНТРАЛЬНЫМ ДЛЯ «ШКОЛЫ ФРОЯНОВА» ЯВЛЯЕТСЯ «НАРОДНОСТЬ» КАК НЕКИЙ ОСНОВОПОЛАГАЮЩИЙ ПРИНЦИП, ПРОТИВОПОСТАВЛЯЮЩИЙ «РУССКОСТЬ» «ЧУЖДЫМ», ПРЕЖДЕ ВСЕГО ЗАПАДНЫМ, ВЛИЯНИЯМ. ПОЭТОМУ ДЛЯ НЕЁ НЕТ НИКАКИХ ПРОТИВОРЕЧИЙ В ОДНОВРЕМЕННОМ ВОЗВЕЛИЧИВАНИИ СЛАВЯНСКОЙ АРХАИКИ (ВПЛОТЬ ДО СИМПАТИЙ К ЯЗЫЧЕСТВУ У ОСНОВАТЕЛЯ «ШКОЛЫ»), «ВЕЧЕВОГО «НАРОДОВЛАСТИЯ», ТИРАНИИ ИВАНА ГРОЗНОГО ИЛИ БОЛЬШЕВИСТСКОЙ (ОСОБЕННО СТАЛИНСКОЙ) ДИКТАТУРЫ. ВСЁ ЭТО БЫЛИ ЯКОБЫ «НАРОДНЫЕ» РЕЖИМЫ И ЯВЛЕНИЯ. И, КОНЕЧНО, СЛЕДОВАЛО БЫ ОГОВОРИТЬСЯ, ЧТО ЭТО НАПРАВЛЕНИЕ НИКОГДА НЕ БЫЛО, И СЕЙЧАС НЕ ЯВЛЯЕТСЯ, МАГИСТРАЛЬНЫМ В РОССИЙСКОМ НОВГОРОДОВЕДЕНИИ И ОТВЕРГАЛОСЬ ПОДАВЛЯЮЩИМ БОЛЬШИНСТВОМ учёных.

БОЛЕЕ СУЩЕСТВЕННО, ОДНАКО, ОПРЕДЕЛЕНИЕ ТИПА НОВГОРОДСКОЙ ГОСУДАРСТВЕННОСТИ, К ЧЕМУ К. ГЁРКЕ ОБРАЩАЕТСЯ В КОНЦЕ КНИГИ. ПО РАЗНЫМ ПРИЧИНАМ И, НА МОЙ ВЗГЛЯД, СОВЕРШЕННО СПРАВЕДЛИВО ОН ОТКАЗЫВАЕТСЯ СЧИТАТЬ НОВГОРОД МОНАРХИЕЙ, БОЯРСКОЙ РЕСПУБЛИКОЙ, ТЕОКРАТИЕЙ ИЛИ (ПО О. СЕВАСТЬЯНОВОЙ) «ПРИВИЛЕГИРОВАННЫМ ВЕЛИКОКНЯЖЕСКИМ ГОРОДОМ». САМ ОН 
ПРЕДЛАГАЕТ ДВА ВАРИАНТА КАК НАИБОЛЕЕ ПРОСТЫЕ: «ГОРОД-ГОСУДАРСТВО» ИЛИ «ВЕЛИКИЙ НОВГОРОД» (САМОНАЗВАНИЕ) (С. 401-402). ВТОРОЕ ОБОЗНАЧЕНИЕ НЕ ИМЕЕТ ОТНОШЕНИЯ К ТИПУ ПОЛИТИЧЕСКОГО ОБРАЗОВАНИЯ (К ТОМУ ЖЕ ПОЯВЛЯЕТСЯ ОНО В ИНТЕРЕСУЮЩЕМ АВТОРА ЗНАЧЕНИИ ТОЛЬКО В ХІV В. ${ }^{17}$ ), А ПЕРВОЕ НЕ ВЫЗЫВАЕТ У МЕНЯ БОЛЬШИХ ВОЗРАЖЕНИЙ. У НЕГО ЕСТЬ, ВПРОЧЕМ, ТОТ НЕДОСТАТОК, ЧТО ОНО ИМЕЕТ АССОЦИАЦИИ, ПРЕЖДЕ ВСЕГО, С АНТИЧНЫМИ ПОЛИСАМИ. МЕЖДУ ТЕМ, НОВГОРОД, ПО МОЕМУ УБЕЖДЕНИЮ, ИМЕЕТ СМЫСЛ СОПОСТАВЛЯТЬ (НО, РАЗУМЕЕТСЯ, НЕ ОТОЖДЕСТВЛЯТЬ) НЕ С НИМИ, А С ЕВРОПЕЙСКИМИ ГОРОДСКИМИ КОММУНАМИ И РЕСПУБЛИКАМИ СРЕДНЕВЕКОВЬЯ И РАННЕГО НОВОГО ВРЕМЕНИ. ЗДЕСЬ НЕ МЕСТО ИЗЛАГАТЬ МОИ ПРЕДСТАВЛЕНИЯ ПО ЭТОМУ ПОВОДУ (В КОНЦЕНТРИРОВАННОМ ВИДЕ ОНИ ИЗЛОЖЕНЫ В ГОТОВЯЩЕЙСЯ К ПУБЛИКАЦИИ МОНОГРАФИИ). ДОСТАТОЧНО ЛИШЬ СКАЗАТЬ, ЧТО ПРИВОДИМЫЕ К. ГЁРКЕ КОНТРАРГУМЕНТЫ НЕ УБЕЖДАЮТ. ВОПРЕКИ ЕГО УТВЕРЖДЕНИЯМ, В НОВГОРОДЕ СУЩЕСТВОВАЛО ВПОЛНЕ ЧЁТКОЕ РАЗЛИЧЕНИЕ МЕЖДУ «ПОЛИТИЧЕСКИМ НАРОДОМ» (ФАКТИЧЕСКИ СОВПАДАВШИМ С КОНЧАНСКИМИ И УЛИЧАНСКИМИ ОБЪЕДИНЕНИЯМИ ГОРОДА НОВГОРОДА) И ВСЕМ ОСТАЛЬНЫМ НАСЕЛЕНИЕМ (ПРЕЖДЕ ВСЕГО СЕЛЬСКИМ). МОЖНО ТОЛЬКО СОГЛАСИТЬСЯ С К. ГЁРКЕ В ТОМ, ЧТО СТРЕМЛЕНИЕ К СВОБОДЕ (das Streben nach Freiheit), ПРИЧЁМ В СМЫСЛЕ «СВОБОДЫ ОТ», БЫЛО ЦЕНТРАЛЬНЫМ ДЛЯ КОММУНАЛЬНОГО ДВИЖЕНИЯ В «ЛАТИНСКОЙ ЕВРОПЕ» (С. 404). НО НЕОЖИДАННЫМ ПРИ ЭТОМ ВЫГЛЯДИТ ЗАЯВЛЕНИЕ АВТОРА, ЧТО «НОВГОРОДСКИЕ ИСТОЧНИКИ НЕ ЗНАЮТ СООТВЕТСТВУЮЩЕЙ ТЕРМИНОЛОГИИ». НО ВЕДЬ КОНЦЕПЦИЯ ВОЛЬНОСТИ В КНЯЗЬЯХ (А ЭТО ИМЕННО И БЫЛА «СВОБОДА ОТ»), А ТАКЖЕ САМООПРЕДЕЛЕНИЕ НОВГОРОДЦЕВ КАК ЩМУЖЕЙ ВОЛЬНЫХ» (ВО ПСКОВЕ - «ДОБРОВОЛЬНЫХ»), БЫЛИ КЛЮЧЕВЫМИ ДЛЯ НОВГОРОДСКОЙ ИДЕОЛОГИИ. И УЖ СОВСЕМ НЕЛЬЗЯ СОГЛАСИТЬСЯ С ИДЕЕЙ К. ГЁРКЕ О ТОМ, ЧТО РАЗВИТИЮ НОВГОРОДА ПО КОММУНАЛЬНОМУ ПУТИ МЕШАЛО ПРАВОСЛАВИЕ. НЕЛЬЗЯ, ЕСТЕСТВЕННО, ОТРИЦАТЬ КОЛОССАЛЬНОЕ ВЛИЯНИЕ ПРАВОСЛАВИЯ НА КУЛЬТУРУ НОВГОРОДА И МИРОСОЗЕРЦАНИЕ НОВГОРОДЦЕВ, НО ОБРАЩЕНИЕ К ИСТОЧНИКАМ, ХАРАКТЕРИЗУЮЩИМ ПОВСЕДНЕВНУЮ ЖИЗНЬ (А НЕ К БОГОСЛОВСКИМ ТРАКТАТАМ), ПОКАЗЫВАЕТ, ЧТО НОВГОРОДЦЫ И МНОГОЧИСЛЕННЫЕ В НОВГОРОДЕ ПРЕДСТАВИТЕЛИ «ЛАТИНСКОЙ ЕВРОПЫ» (ГАНЗЕЙСКИЕ КУПЦЫ В XIII-XV ВВ., А ДО ЭТОГО СКАНДИНАВЫ) ПРЕКРАСНО ПОНИМАЛИ ДРУГ ДРУГА И СЧИТАЛИ ДРУГ ДРУГА ХРИСТИАНАМИ. ГАНЗЕЙСКИЕ КУПЦЫ БРАЛИ БЛАГОСЛОВЕНИЕ У НОВГОРОДСКИХ АРХИЕПИСКОПОВ, А ТЕ ВЫСТУПАЛИ ПОСРЕДНИКАМИ В ИХ КОНФЛИКТАХ С НОВГОРОДСКИМИ ПАРТНЁРАМИ ${ }^{18}$. ПРИНАДЛЕЖНОСТЬ НОВГОРОДА К ВИЗАНТИЙСКО-ПРАВОСЛАВНОМУ КУЛЬТУРНОМУ КРУГУ, КОНЕЧНО, МОГЛА ОКАЗЫВАТЬ ВОЗДЕЙСТВИЕ НА ПОЛИТИЧЕСКИЙ ЯЗЫК И ИДЕОЛОГИЮ, НО ВРЯД ЛИ СТОИТ - ВСЛЕД ЗА НАЦИОНАЛ-РОМАНТИКАМИ - СЧИТАТЬ ЕГО ОСНОВОЙ НЕКОЕГО Sonderweg (ИЛИ, КАК ПИШЕТ САМ АВТОР, Sonderform, c. 405). ВО ВСЯКОМ СЛУЧАЕ, ИСТОЧНИКАМИ ТАКИЕ ПРЕДСТАВЛЕНИЯ НЕ ПОДТВЕРЖДАЮТСЯ, ХОТЯ, РАЗУМЕЕТСЯ, К. ГЁРКЕ ПРАВ В ТОМ, ЧТО В НОВГОРОДЕ НЕ СУЩЕСТВОВАЛО ЛАТИНСКОЙ (РИМСКОЙ) ПРАВОВОЙ ТРАДИЦИИ В ТЕХ ФОРМАХ, В КОТОРЫХ ОНА СПОСОБСТВОВАЛА Verrechtlichung В ЗАПАДНОЙ ЕВРОПЕ, И НОВГОРОДЦАМ ПРИХОДИЛОСЬ ИСКАТЬ СВОИ ПОНЯТИЯ ДЛЯ ОБОЗНАЧЕНИЯ РЕАЛИЙ, ВО МНОГОМ БЛИЗКИХ К ЗАПАДНОЕВРОПЕЙСКИМ. НО ТАКИЕ ПОНЯТИЯ ВОЗНИКАЛИ (НАПРИМЕР, «ВЕСЬ НОВГОРОД» КАК СВОЕОБРАЗНЫЙ АНАЛОГ ЛАТИНСКОГО commune/communitas) ${ }^{19}$. ЕЩЁ РАЗ ПОДЧЕРКНУ: РЕСПУБЛИКАНСКАЯ (КОММУНАЛЬНАЯ) МОДЕЛЬ РЕЛЕВАНТНА ДЛЯ НОВГОРОДА ИМЕННО КАК ИССЛЕДОВАТЕЛЬСКАЯ МОДЕЛЬ; ТЕ КОНКРЕТНЫЕ ОСОБЕННОСТИ НОВГОРОДСКОГО 
ТИПА ПОЛИТИЧЕСКОГО УСТРОЙСТВА, НА КОТОРЫХ АКЦЕНТИРУЕТ ВНИМАНИЕ К. ГЁРКЕ (НАПРИМЕР, ОТСУТСТВИЕ ИЛИ, ТОЧНЕЕ, НЕРАЗВИТОСТЬ ГОРОДСКОГО СОВЕТА), ДЕЙСТВИТЕЛЬНО ИМЕЛИ МЕСТО И ДОЛЖНЫ БЫТЬ ОСМЫСЛЕНЫ. В КОНЕЧНОМ СЧЁТЕ, ВСЯ ДИСКУССИЯ СВОДИТСЯ К ИЗВЕСТНОМУ (И ВЕЧНОМУ) СПОРУ О НАПОЛОВИНУ ПУСТОМ ИЛИ ПОЛНОМ СТАКАНЕ. САМ АВТОР В КОНЦЕ КОНЦОВ ОТМЕЧАЕТ, ЧТО НОВГОРОД БЫЛ НЕОТЪЕМЛЕМОЙ ЧАСТЬЮ ДРЕВНЕРУССКОЙ «ГОРОДСКОЙ КОНЦЕПЦИИ» И В ТО ЖЕ ВРЕМЯ БОЛЬШЕ ПОХОЖ НА «ЛАТИНСКОЕВРОПЕЙСКУЮ МОДЕЛЬ», ЧЕМ НА МОСКОВСКОЕ ГОСУДАРСТВО (ЧЕМУ, КАК ВИДИМ, НИКАК НЕ ПОМЕШАЛО ПРАВОСЛАВИЕ). И С ТЕМ, И С ДРУГИМ МОЖНО ТОЛЬКО СОГЛАСИТЬСЯ, А К. ГЁРКЕ НУЖНО ПОЗДРАВИТЬ С УСПЕШНОЙ РЕАЛИЗАЦИЕЙ ТОЙ СЛОЖНОЙ ЗАДАЧИ, КОТОРУЮ ОН ПЕРЕД СОБОЙ ПОСТАВИЛ. СДЕЛАННЫЕ ВЫШЕ ЗАМЕЧАНИЯ НОСЯТ В ОСНОВНОМ ДИСКУССИОННЫЙ, А НЕ УЗКО-КРИТИЧЕСКИЙ ХАРАКТЕР, А НОВГОРОДСКАЯ ТЕМА СТОЛЬ НЕОБЪЯТНА, ЧТО ОСВЕТИТЬ ЕЁ ПОЛНОСТЬЮ В ОДНОЙ МОНОГРАФИИ НЕВОЗМОЖНО, И ВЫБОР АКЦЕНТОВ ВСЕГДА В ТОЙ ИЛИ ИНОЙ МЕРЕ СУБЪЕКТИВЕН. НО ПРОФЕССОРУ КАРСТЕНУ ГЁРКЕ УДАЛОСЬ, КАК КАЖЕТСЯ, ДОСТИЧЬ ПОЧТИ ИДЕАЛЬНОГО БАЛАНСА.

\section{NOTES}

1. C. Goehrke, «Gross-Novgorod und Pskov/Pleskau», in M. Hellmann, K. Zernack, G. Schramm, hrsg., Handbuch der Geschichte Russlands, vol. 1, Stuttgart: Hiersemann, 1981, p. 431-483.

2. В.Л. ЯНИН, ОЧЕРКИ ИСТОРИИ СРЕДНЕВЕКОВОГО НОВГОРОДА М.: ЯЗЫКИ СЛАВЯНСКИХ КУЛЬТУР, 2008; Ph. Frison et O. Sevastyanova, eds., Novgorod ou la Russie oubliée. Une république commerçante (XII ${ }^{e}-\mathrm{XV}^{e}$ siècles), Charenton-le-Pont : Le Ver à Soie, 2015.

3. Я.С. ЛУРЬЕ, ДВЕ ИСТОРИИ РУСИ ХV ВЕКА. РАННИЕ И ПОЗДНИЕ, НЕЗАВИСИМЫЕ И ОФИЦИАЛЬНЫЕ ЛЕТОПИСИ ОБ ОБРАЗОВАНИИ МОСКОВСКОГО ГОСУДАРСТВА, СПБ.: ДМИТРИЙ БУЛАНИН, 1994, С. 142-143.

4. Б.Н. ФЛОРЯ, “ПРИЗВАНИЕ РЮРИКА И ОСНОВАНИЕ НОВГОРОДА”, ВЕСТНИК МОСКОВСКОГО УНИВЕРСИТЕТА, СЕР. 8 «ИСТОРИЯ», 2012, № 5, С. 3-9.

5. А.А. ГИППИУС, «НОВГОРОДСКАЯ ВЛАДЫЧНАЯ ЛЕТОПИСЬ ХІІ-XIV ВВ. И ЕЁ АВТОРЫ (ИСТОРИЯ И СТРУКТУРА ТЕКСТА В ЛИНГВИСТИЧЕСКОМ ОСВЕЩЕНИИ», in ЛИНГВИСТИЧЕСКОЕ ИСТОЧНИКОВЕДЕНИЕ И ИСТОРИЯ РУССКОГО ЯЗЫКА 2004-2005 М., 2006, с. 114-251; А.А. ГИППИУС, «АРХИЕПИСКОП АНТОНИЙ, НОВГОРОДСКОЕ ЛЕТОПИСАНИЕ И КУЛЬТ СВЯТОЙ СОФИИ», in А.Е. МУСИН, СОСТ., ХОРОШИЕ ДНИ. ПАМЯТИ А.С. ХОРОШЕВА, ВЕЛИКИЙ НОВГОРОД - СПБ. - М., 2009, с. 181-198.

6. M. Monk, P. Johnston, «Perspectives on Non-wood Plants in the Sampled Assemblage from the Troitsky Excavations of Medieval Novgorod», in M.A. Brisbane, N.A. Makarov, E.N. Nosov, eds., The Archaeology of Medieval Novgorod in Context. Studies in Centre/Periphery Relations, Oxford: Oxbow Books, 2012, p. 283-320; A. Alsleben, «The Plant Economy of Northern Medieval Russia», in The Archaeology of Medieval Novgorod, p. 321-350.

7. L.K. Goetz, Deutsch-Russische Handelsgeschichte des Mittelalters, Lübeck: Otto Waelde, 1922 (Hansische Geschichtsquellen, Neue Folge, vol.5), p.316-319; А.Л. ХоРОшкЕВич, ТоРГОВлЯ ВЕЛИКОГО НОВГОРОДА С ПРИБАЛТИКОЙ И ЗАПАДНОЙ ЕВРОПОЙ В XIV-XV ВЕКАХ, М.: ИЗДАТЕЛЬСТВО АКАДЕМИИ НАУК СССР, 1963, С. 323-324.

8. А.П. ТОЛОЧКО, «ИСТОРИЯ РОССИЙСКАЯ» ВАСИЛИЯ ТАТИЩЕВА: ИСТОЧНИКИ И ИЗВЕСТИЯ КИЕВ: КРИТИКА, 2005, с. 196-245. 
9. ПОЛНОЕ СОБРАНИЕ РУССКИХ ЛЕТОПИСЕЙ Т. 3: НОВГОРОДСКАЯ ЛЕТОПИСЬ СТАРШЕГО И МЛАДШЕГО ИЗВОДОВ, М.: ЯЗЫКИ РУССКОЙ КУЛЬТУРЫ, 2000, С. 174 (ДАЛЕЕ: ПСРЛ, Т. 3).

10. В.А. КУЧКИН, «ДЕСЯТСКИЕ И СОТСКИЕ ДРЕВНЕЙ РУСИ», in А.А. ГОРСКИЙ И ДР., ДРЕВНЯЯ РУСЬ: ОЧЕРКИ ПОЛИТИЧЕСКОГО И СОЦИАЛЬНОГО СТРОЯ М.: ИНДРИК, 2008, С. 270-425; В.А. КУЧКИН, «ТЫСЯЦКИЕ В НОВГОРОДЕ В ДОМОНГОЛЬСКИЙ ПЕРИОД» іn ТВЕРЬ, ТВЕРСКАЯ ЗЕМЛЯ И СОПРЕДЕЛЬНЫЕ ТЕРРИТОРИИ В ЭПОХУ СРЕДНЕВЕКОВЬЯ, ВЫП. 10, ТВЕРЬ, 2017, С. 6-16.

11. А.А. ГИППИУС, «СОПЕРНИЧЕСТВО ГОРОДСКИХ КОНЦОВ КАК ФАКТОР КУЛЬТУРНОЙ ИСТОРИИ НОВГОРОДА ХІІ-ХІІІ ВВ.», in М.М. КРОМ, РЕД., СПОРЫ О НОВГОРОДСКОМ ВЕЧЕ: МЕЖДИСЦИПЛИНАРНЫЙ ДИАЛОГ, СПБ.: ЕВРОПЕЙСКИЙ УНИВЕРСИТЕТ В САНКТ-ПЕТЕРБУРГЕ, 2012, С. 121-136.

12. А.А. ГОРСКИЙ, ДРЕВНЕРУССКАЯ ДРУЖИНА (К ИСТОРИИ ГЕНЕЗИСА КЛАССОВОГО ОБЩЕСТВА И ГОСУДАРСТВА НА РУСИ), М.: ПРОМЕТЕЙ, 1989, С. 44-45.

13. М.Х. АЛЕШКОВСКИЙ, «СОЦИАЛЬНЫЕ ОСНОВЫ ФОРМИРОВАНИЯ ТЕРРИТОРИИ НОВГОРОДА IXXV ВЕКОВ ", СОВЕТСКАЯ АРХЕОЛОГИЯ, 1974, № 3, С. 104-105.

14. ПСРЛ, Т. 3, С. 82-84.

15. А.А. ГИППИУС, НОВГОРОДСКАЯ ВЛАДЫЧНАЯ ЛЕТОПИСЬ, С. 214-215.

16. НАЧИНАЯ С КЛАССИЧЕСКОЙ: В.И. СЕРГЕЕВИЧ, ВЕЧЕ И КНЯЗЬ. РУССКОЕ ГОСУДАРСТВЕННОЕ УСТРОЙСТВО И УПРАВЛЕНИЕ ВО ВРЕМЕНА КНЯЗЕЙ РЮРИКОВИЧЕЙ. ИСТОРИЧЕСКИЕ ОЧЕРКИ М.: ТИПОГРАФИЯ А.В. МАМОНТОВА, 1867.

17. П.В. лУКИН, «ВЕЛИкий НовгоРод», Slověne. International Journal of Slavic Studies, 2018, 7, № 2, c. $383-413$.

18. П.В. ЛУКИН, «ПОЛИТИЧЕСКИЕ ФУНКЦИИ НОВГОРОДСКИХ АРХИЕПИСКОПОВ В ГАНЗЕЙСКИХ ДОКУМЕНТАX XIV-XV ВВ.», in ЦЕРКОВЬ В ИСТОРИИ РОССИИ. СБОРНИК 13: К 90-ЛЕТИЮ СО ДНЯ РОЖДЕНИЯ ЧЛ.-КОРР. РАН Я.Н. ЩАПОВА, М: ИНСТИТУТ РОССИЙСКОЙ ИСТОРИИ РАН, 2020, С. 97-127.

19. П.В. ЛУКИН, «“ВЕСЬ НОВГОРОД”: К ВОПРОСУ О ПРОИСХОЖДЕНИИ И СОДЕРЖАНИИ ПОНЯТИЯ», РОССИЙСКАЯ ИСТОРИЯ, 2020, № 4, С. 56-63.

\section{AUTEURS}

\section{PAVEL LUKIN}

Institut d'histoire de Russie, Académie des sciences de Russie Académie russe de l'économie nationale et du service public 